\title{
Broadening the biocompatibility of gold nanorods from rat to Macaca fascicularis: advancing clinical potential
}

Jinfeng Liao ${ }^{1 \dagger}$, Taorang Tian ${ }^{1 \dagger}$, Sirong Shi ${ }^{1}$, Xueping Xie ${ }^{1}$, Shuanglin Peng ${ }^{2}$, Ying Zhu ${ }^{3,4^{*}}$, Jingang Xiao ${ }^{2^{*}}$ and Yunfeng $\operatorname{Lin}^{1,5^{*}}$ (D)

\begin{abstract}
Background: The biomedical field has used gold nanorods (GNRs) for decades; however, clinical trials and translation is limited except gold nanoshells. The preparation of gold nanoshells is more complex than that of polyethylene glycol-modified GNRs (PEG-GNRs), and it is difficult to ensure uniform thickness. It is important to encourage and broaden the use of the star member (PEG-GNRs) of gold nanoparticles family for clinical translation. Existing studies on PEG-GNRs are limited with no relevant systematic progression in non-human primates. Herein, we assessed the systematic biocompatibility of PEG-GNRs in rats and clinically relevant Macaca fascicularis.
\end{abstract}

Results: In this small animal study, we administrated multiple doses of PEG-GNRs to rats and observed good biocompatibility. In the non-human primate study, PEG-GNRs had a longer blood half-life and produced a negligible immune response. Histological analysis revealed no significant abnormality.

Conclusions: PEG-GNRs were well-tolerated with good biocompatibility in both small animals and large non-human primates. The information gained from the comprehensive systemic toxicity assessment of PEG-GNRs in M. fascicularis will be helpful for translation to clinical trials.

Keywords: Gold nanorods, Systemic biocompatibility, Macaca fascicularis, Blood clearance, Biodistribution, Major organ analysis

\section{Introduction}

Gold nanoparticles are undergoing rapid development,

*Correspondence: zhuying@zjlab.org.cn; drxiaojingang@163.com; yunfenglin@scu.edu.cn

${ }^{\dagger}$ Jinfeng Liao and Taorang Tian contributed equally to this work

1 State Key Laboratory of Oral Diseases, National Clinical Research Centre

for Oral Diseases, West China Hospital of Stomatology, Sichuan University,

Chengdu 610041, China

${ }^{2}$ Department of Oral and Maxillofacial Surgery, The Affiliated

Stomatology Hospital of Southwest Medical University, Luzhou 646000,

China

${ }^{3}$ Division of Physical Biology, CAS Key Laboratory of Interfacial Physics and Technology, Shanghai Synchrotron Radiation Facility,

Shanghai Institute of Applied Physics, Chinese Academy of Sciences, Shanghai 201800, China

Full list of author information is available at the end of the article and their unique properties give them great potential for clinical applications such as drug carriers, diagnostic tools, and therapeutic agents. A clinical pilot study employed gold nanoshells in a photothermal therapy approach to treat prostate tumors [1]. Gold nanoparticles are promising in clinical and biomedical applications, such as gold nanorods (GNRs) as nanocarriers and photothermal agents. Murphy's group first invented and synthesized GNRs [2, 3]. GNRs can be prepared via a well-established, reproducible seed-mediated route. GNRs are simple to produce, and they possess long-time colloidal stability. However, the residual cetyltrimethylammonium bromide (CTAB) used as a template during GNRs synthesis is highly toxic [4]. The residual CTAB needs to be replaced to improve the biocompatibility of

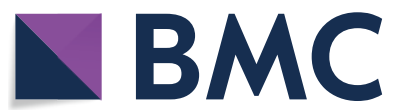

(c) The Author(s) 2021. This article is licensed under a Creative Commons Attribution 4.0 International License, which permits use, sharing, adaptation, distribution and reproduction in any medium or format, as long as you give appropriate credit to the original author(s) and the source, provide a link to the Creative Commons licence, and indicate if changes were made. The images or other third party material in this article are included in the article's Creative Commons licence, unless indicated otherwise in a credit line to the material. If material is not included in the article's Creative Commons licence and your intended use is not permitted by statutory regulation or exceeds the permitted use, you will need to obtain permission directly from the copyright holder. To view a copy of this licence, visit http://creativeco mmons.org/licenses/by/4.0/. The Creative Commons Public Domain Dedication waiver (http://creativecommons.org/publicdomain/ zero/1.0/) applies to the data made available in this article, unless otherwise stated in a credit line to the data. 
GNRs [5, 6]. Polyethylene glycol (PEG) is a classic candidate to replace CTAB for GNRs modification $[7,8]$ and PEG have been used in clinical translation under the approval the Food and Drug Administration. PEG modification is a potential strategy for improving stability, reducing toxicity, and enhancing membrane transport $[9,10]$. GNRs are rod-shaped, with unique longitudinal and transverse surface plasmon resonance $[11,12]$ as well as an adjustable aspect ratio which allows near-infrared region (NIR) absorption [13, 14]. These properties confer two unique advantages to PEG-modified GNRs (PEGGNRs): 1) their synthesis is easier and more controllable than that of gold nanoshell and 2) their NIR adsorption is adjustable by their aspect ratios. PEG-GNRs have multiple scientific applications [15] such as photothermal therapy agents, [16-20] bioimaging agents, [21-24] drug carriers, [25-27] gene delivery agents, [28, 29] and biosensors [30-32]. These continued developments give great potential for their clinical use. Unfortunately, PEGGNRs research has remained theoretical, and no preclinical studies in large animals exist. Different shapes of PEG-GNRs and gold nanoshells may produce different responses in the body. Thus, studies on the matter of PEG-GNRs are required.

Previous in vivo toxicity studies of PEG-GNRs have focused on rodents, because numerous nanomedicines have been used successfully in small animals but failed in clinical practice, [31] and the toxicity results from small animals alone are not reliable. The systemic toxicity of some heavy metal and organic nanoparticles such as CdSe/CdS/ZnS quantum dots, [34, 35] silica quantum dots, [36] detonation nanodiamonds, [37] and graphene oxide [38] have been studied in large animals. In one recent study, anaphylactic death of non-human primates occurred with graphene oxide but not with single-walled carbon nanotubes and nanodiamonds [39]. These studies highlight the great importance of assessing nanotoxicity in large animals. A broadening systemic understanding of biocompatibility in non-primate animals is desperately needed to bring GNRs into clinical research.

In this study, we systemically assessed the toxicity of PEG-GNRs in rat and Macaca fascicularis, because of their genetic similarity to humans. We treated M. fascicularis with PEG-GNRs and assessed their blood pharmacokinetic parameters, biodistribution, blood immune response, urinalysis, and histology. We observed no significant changes between the PEG-GNRs-treated group and control group, except a negligible immune response that was well-tolerated. Histological analysis revealed no severe toxicity. The remaining inorganic nanomaterial in the reticuloendothelial system (RES) showed low shortterm breakdown and clearance of PEG-GNRs. The biocompatibility assessment in higher mammals furthers our understanding of the biosafety of PEG-GNRs, which may support the development of their clinical application.

\section{Results and discussion}

There are four steps in the research and development of new nanomedicine agents: discovery, preclinical research, clinical research, and new drug declaration. The role of preclinical research is to ensure the nontoxicity of compounds before starting human trials [37]. For further development of gold nanorods, we assessed their systemic biocompatibility in rodents and non-primate animals. Figure 1a shows how the prepared PEG-GNRs were injected in rats and M. fascicularis and biocompatibility was assessed in blood, tissue, and urine samples. Our detailed experimental design is shown in Fig. 1b. Studies in the Spraque-Dawley (SD) rats included pharmacokinetic clearance, standard blood measures, and major organ biocompatibility. The studies in $M$. fascicularis included blood measures, urinalysis, and measures of body mass, body temperature, blood pressure, and tissue distributions at designated time points.

We prepared the PEG-GNRs in two steps. After synthesizing the CTAB-GNRs using a modified seed-mediated approach, we PEGylated them with PEG-SH (Fig. 2a). The prepared CTAB-GNRs possess an aspect ratio of approximately 3.9 (length: $\sim 47 \mathrm{~nm}$, width: $\sim 12 \mathrm{~nm}$ ), as shown in the transmission electron microscopy (TEM) image (Fig. 2b). Replacing CTAB with PEG-SH via $\mathrm{Au}-\mathrm{S}$ bonds formation yielded the PEG-GNRs. In Fig. 2c, the PEG coating is visible as a faint halo around the GNRs, which is not present on CTAB-GNRs. The color of their dispersions was not different (inset photographs in Fig. 2b, c). The atomic force microscopy (AFM) images also demonstrate the morphology of CTAB-GNRs (Fig. 2d) and PEGGNRs (Fig. 2e). The maximum absorption wavelengths of the CTAB-GNRs and PEG-GNRs were in the NIR region, at approximately $800 \mathrm{~nm}$ and $805 \mathrm{~nm}$, respectively (Fig. 2f). The zeta potential of PEG-GNRs was $-1.2 \mathrm{mV}$, whereas that of CTAB-GNRs was at $+39.6 \mathrm{mV}$ (Fig. 2g). The replacement of CTAB with PEG greatly altered the surface charge.

Next, we assessed the systemic toxicity of PEG-GNRs in rats and monkeys. Figure 3 a shows the blood clearance, tissue distribution, and organ toxicity of PEG-GNRs in rats. As shown in Fig. 3b, blood markers and hepatic/ renal function indicators were within normal range, with no substantial differences between the treated and control groups.

To quantitatively assess the blood circulation time and biodistribution of PEG-GNRs, we measured the remaining amount of gold $(\mathrm{Au})$ in blood samples and tissues via inductively coupled plasma-atomic emission 
spectroscopy (ICP-AES). PEG-GNRs cleared from the blood with a longer half-life $\left(t_{1 / 2}=\sim 53 \mathrm{~min}\right)$ (Fig. 3c) than that of CTAB-GNRs $\left(\mathrm{t}_{1 / 2}<15 \mathrm{~min}\right)$ cleared from the blood [40-42]. After $24 \mathrm{~h}$, there was no residual Au in the blood. We evaluated the biodistribution of PEG-GNRs in rats at 3 days, 1 week, and 2 weeks after the treatment. Our results show that PEG-GNRs were mainly localized in the spleen and liver (Fig. 3d and Additional file 1: Figure S1). Although some doubts remain regarding the distribution of gold nanorods in the RES, [43-45] because the spleen is the major part of the RES, it can sequester foreign compounds entering the body. The concentration of $\mathrm{Au}$ in the spleen was almost 3.5-fold (average of the three test groups) higher than in the liver, and the relative concentrations of $\mathrm{Au}$ in the spleen and liver decreased with increasing doses. The ratios of Au in the spleen and liver were approximately $3.9,3.5$, and 3.0 for a dose of 5,10 , and $20 \mathrm{mg} / \mathrm{kg}$, respectively. We hypothesize that when the spleen reaches saturation, the remaining $\mathrm{Au}$ is selectively distributed to the liver.

Although PEG-GNRs are mainly distributed in the RES histological analysis revealed no apparent structural change or abnormality in the spleen and liver (Fig. 3e and Additional file 1: Figure S2). Examination of the heart, lungs, and kidneys also showed no structural alteration and confirmed the absence of toxicity in vivo. No mortality and adverse effects on behavior occurred in the animals, even when treated with a maximal dose of $20 \mathrm{mg} /$ kg PEG-GNRs.

Next, we conducted a pilot study of PEG-GNRs in $M$. fascicularis (Fig. 4a). The experimental group $(\mathrm{n}=3)$ was administered PEG-GNRs $(1.0 \mathrm{mg} / \mathrm{kg})$ by intravenous drip over $30 \mathrm{~min}$. Peripheral blood samples were collected at designated time points. As shown in Fig. 4b, the white blood cell (WBC) count increased during the first $12 \mathrm{~h}$ but did not exceed the normal range. Within 2 days after injection, alanine aminotransferase (ALT) and aspartate aminotransferase (AST) levels increased. Then, at 3 days, levels were restored to the normal range compared with the control group (gray boxes) and literature reference values (light gray boxes). WBCs increased within 1 day, and then gradually returned to normal levels. These results suggest that PEG-GNRs induced a slight immune response in the early stage after treatment. Because

\section{$\mathbf{a}$}

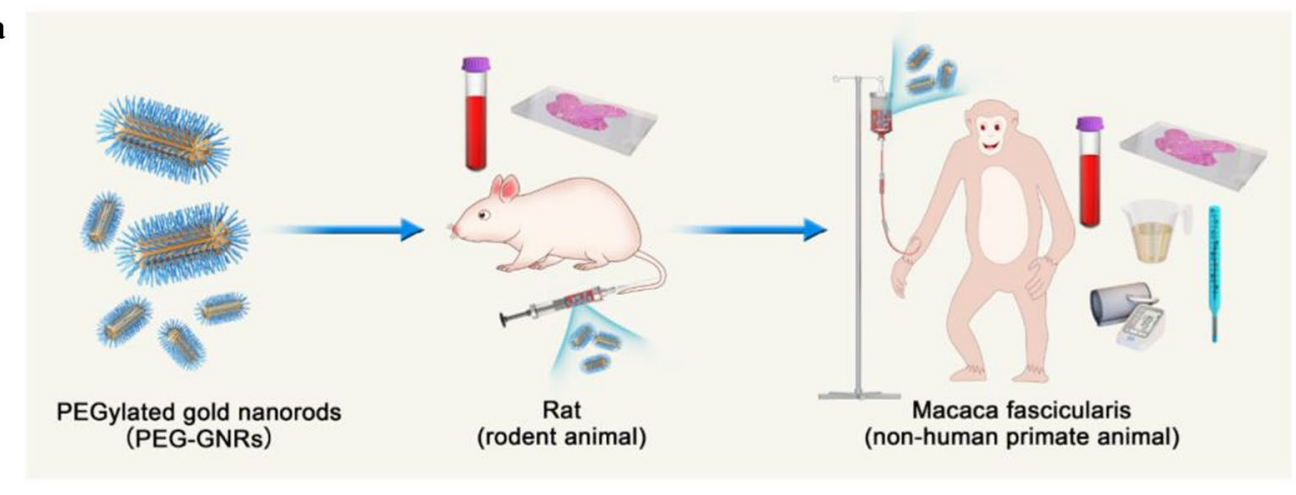

$\mathbf{b}$

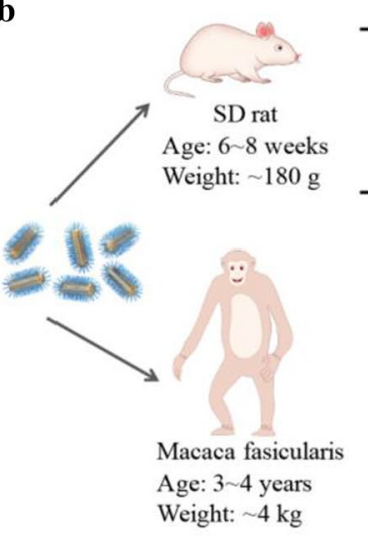

Groups

Test

Control $(\mathrm{n}=3)$

$5 \mathrm{mg} / \mathrm{kg}(\mathrm{n}=3)$

$10 \mathrm{mg} / \mathrm{kg}(\mathrm{n}=3)$

$20 \mathrm{mg} / \mathrm{kg}(\mathrm{n}=3)$

Blood clearance: 0.25 h, 0.75 h, 1 h, 2 h, 6 h, 12 h, 24 h, 48 h

Blood routine test: 3 days, 1 week, 2 weeks

Major organs biodistribution and biocompatibility: 2 weeks

Blood clearance: $1 \mathrm{~min}, 10 \mathrm{~min}, 20 \mathrm{~min}, 30 \mathrm{~min}, 45 \mathrm{~min}, 1 \mathrm{~h}, 2 \mathrm{~h}$, 4 h, 6 h, 8 h, 12 h, 24 h, 48 h

Blood test: $1 \mathrm{~h}, 2 \mathrm{~h}, 6 \mathrm{~h}, 12 \mathrm{~h}, 1$ day, 2 days, 3 days and each week

Urine analysis: every week

Body mass: every week

Body temperature: every week

Blood pressure: every week

Biodistribution (Major organ): 12 weeks

Biocompatibilty analysis: 12 weeks (heart, liver, spleen, lung,

kidney, brain, lymph, intestine, skeleton muscle and skin)

Fig. 1 a The systemic biocompatibility study on PEG-GNRs in rats and Macaca fasicularis. $\mathbf{b}$ Experiment design 
a

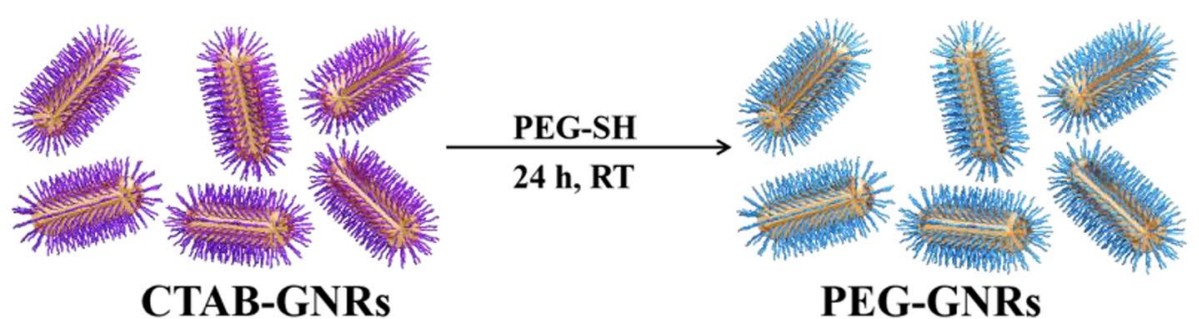

CTAB-GNRs

b
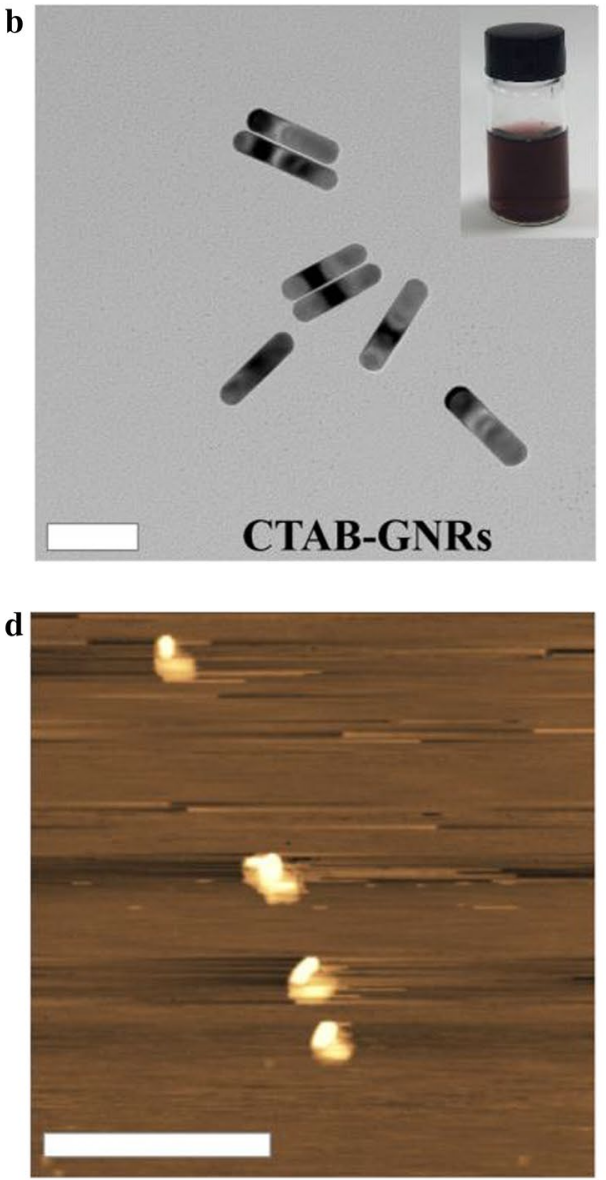

f

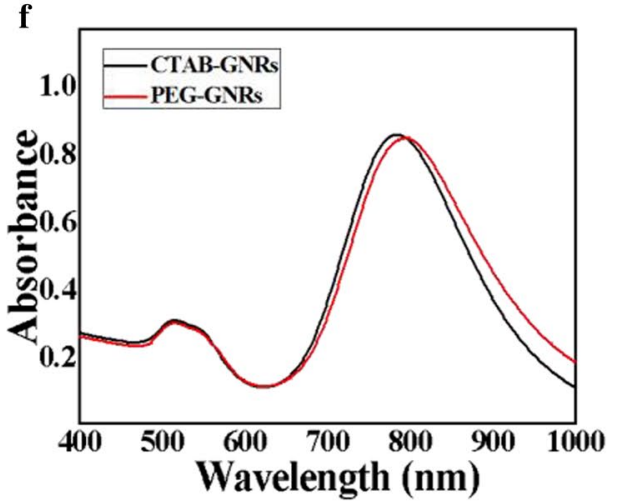

c

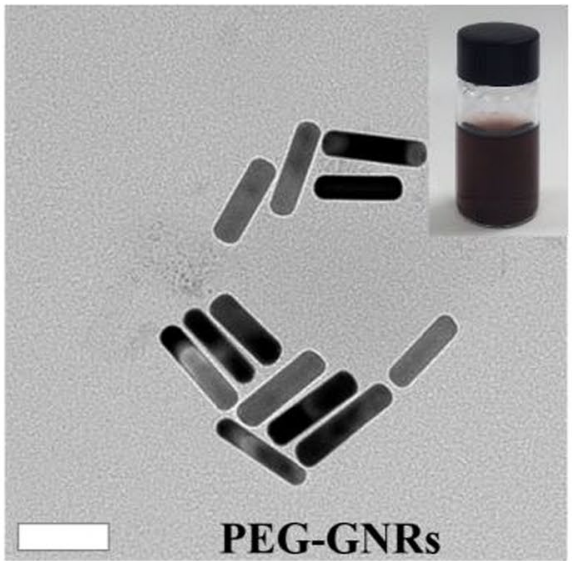

e

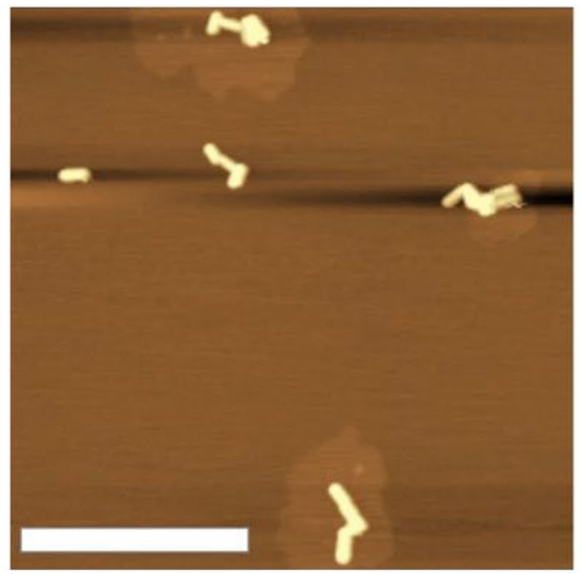

g

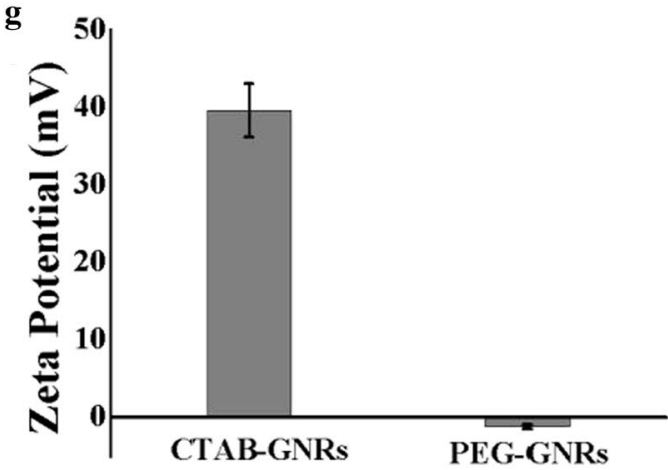

Fig. 2 a The PEG-GNRs synthesis scheme. TEM images of (b) CTAB-GNRs and $\mathbf{c}$ PEG-GNRs. (Scale bars $=50 \mathrm{~nm}$. The inset pictures are the photographs of CTAB-GNRs and PEG-GNRs.) AFM images of (d) CTAB-GNRs and e PEG-GNRs. (Scale bars $=500 \mathrm{~nm}$ ) (f) UV-Vis absorbance spectra of CTAB-GNRs and PEG-GNRs. $\mathbf{g}$ Zeta potentials of CTAB-GNRs and PEG-GNRs 
GNRs are most likely identified as foreign elements, an increase in WBC was expected. ALT increased but stayed within the normal range for 2 days. AST increased beyond the normal range, but the difference was not significant. Slight changes in these markers suggest that the liver acts as an important detoxification organ to tolerate PEG-GNRs [46]. The other kidney function and blood markers (such as neutrophils, lymphocytes, monocytes, eosinophils, and basophils: Additional file 1: Figure S3) were within normal ranges. Although a minor immune response occurred early after injection, we observed no apparent signs of allergic or toxic reactions.

To determine the blood clearance of PEG-GNRs, we collected blood samples at designated time points. Figure $4 \mathrm{c}$ shows the ratio of remaining $\mathrm{Au}$ in the blood clearance profile. The Au blood half-life was approximately $84 \mathrm{~min}$. At $12 \mathrm{~h}$ post-injection, the remaining amount of $\mathrm{Au}$ in the blood reached $3.89 \%$. The blood half-life of PEG-GNRs in $M$. fascicularis was longer than that in the rat. We also determined the remaining concentrations of $\mathrm{Au}$ in the major organs 12 weeks after injection and found PEG-GNRs accumulated predominantly in the spleen and liver (Fig. 4d), similar to the results in the rodent. Next, we collected the major organs and tissues such as heart, liver, spleen, lung, kidney, brain, lymph, intestine, skeleton muscle, and skin for hematoxylin and eosin (H\&E) histological staining sections (Fig. 4e and Additional file 1: Figures S4, Figure S5). We found no significant changes in these organs, even in the liver and spleen, where the PEG-GNRs accumulated and thus observed no signs of PEG-GNRs-induced tissue damage. According to previous studies, [47-49] the main route of nanoparticles clearance is through the extravascular extracellular space (EES), and the small particles $(<9 \mathrm{~nm})$ are eliminated through the kidney. Based on biodistribution results, PEG-GNRs clearance might occur gradually through the EES [50, 51]. Although PEG-GNRs showed no toxicity in rats and $M$. fascicularis, long-term (1 or 2 year) studies are needed to determine their ultimate elimination route and satisfy clinical safety concerns $[52$, 53].

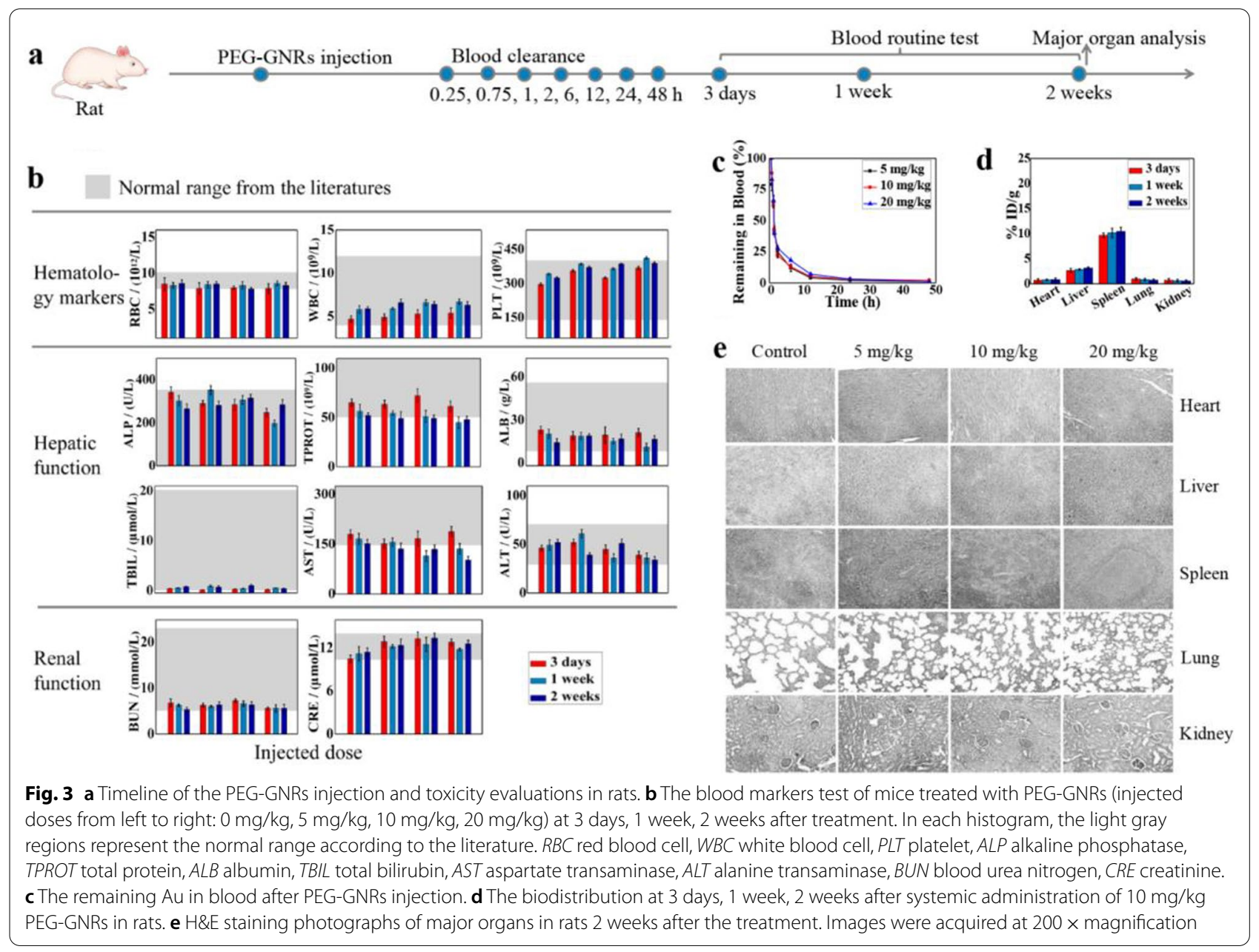


Next, we performed urinalysis in control (Table 1) and experimental animals (Tables 2, 3, and 4). All urine markers were within the normal range and the urine samples were yellow and clear, suggesting functional bladder and kidneys. We monitored other body signs such as body temperature, body weight, and blood pressure weekly (Fig. 5). There were no instances of fever or abnormal temperature. Body weight increased gradually over time, and blood pressure remained almost within the normal range. The animals' activities were supervised and recorded throughout the 12-week evaluation period and showed no abnormal behaviors or vomiting. Thus, the results indicate that PEG-GNRs were relatively safe and well-tolerated in a non-human primate animal.

\section{Materials and methods}

\section{Materials}

Chloroauric acid $\left(\mathrm{HAuCl}_{4} \cdot 3 \mathrm{H}_{2} \mathrm{O}\right)$ and cetyltrimethylammonium bromide (CTAB) were purchased from Sinopharm Chemical Reagent (Shanghai, China) and China National Medicines (Beijing, China), respectively. Thiolated-PEG (mPEG $\left.5000^{-} \mathrm{SH}\right)$ was purchased from Sigma-Aldrich (USA). L-ascorbic acid (Vc) and silver nitrate $\left(\mathrm{AgNO}_{3}\right)$ were purchased from Alfa Chemicals (UK). Sulfuric acid $\left(\mathrm{H}_{2} \mathrm{SO}_{4}\right)$, nitric acid $\left(\mathrm{HNO}_{3}\right)$, hydrochloric acid $(\mathrm{HCl})$, and perchloric acid $\left(\mathrm{HClO}_{4}\right)$ were obtained from KeLong Chemicals Reagent (Chengdu, China). All reagents were of analytical grade.

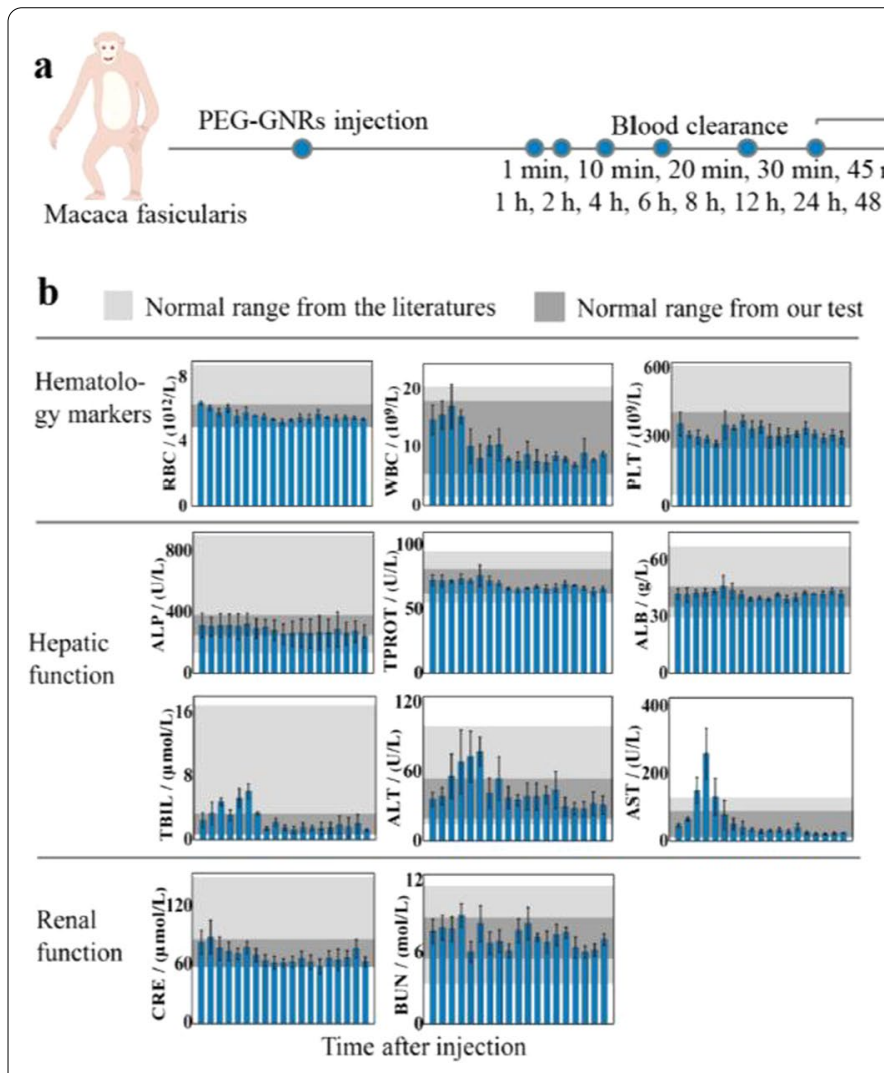

Physical examination: blood routine,

urine analysis, body mass, etc.

Major organ analysis Each week

1 week

12 weeks

$1 \mathrm{~min}, 10 \mathrm{~min}, 20 \mathrm{~min}, 30 \mathrm{~min}, 45 \mathrm{~min}$
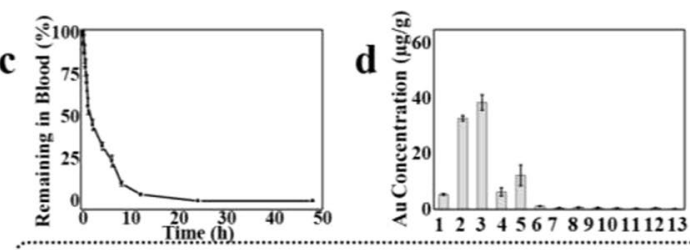

1, Heart; 2, Liver, 3, Spleen, 4, Lung; 5, Kidney; 6, Lymph; 7. Thymus; 8, Brain; 9, Muscle; 10, Skin; 11, Testis; 12, Intestinal; 13, Bone marrow
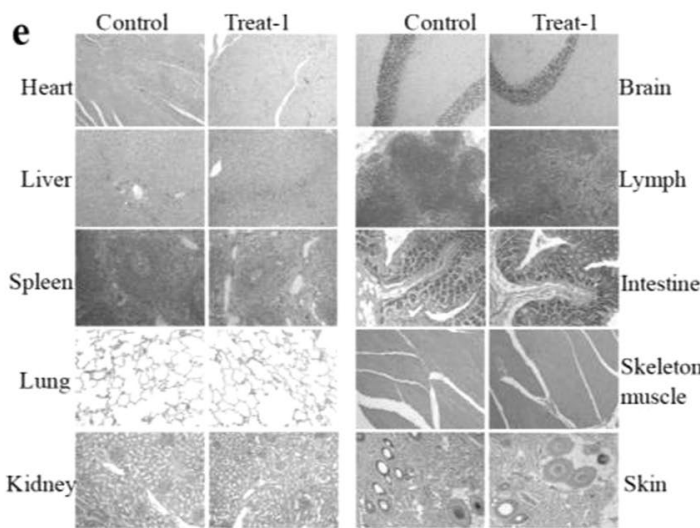

Fig. 4 a Timeline of the PEG-GNRs injection and toxicity evaluations in Macaca fascicularis. $\mathbf{b}$ Blood test results. Times after injection in the figures from left to right are $1 \mathrm{~h}, 2 \mathrm{~h}, 6 \mathrm{~h}, 12 \mathrm{~h}, 1 \mathrm{~d}, 2 \mathrm{~d}, 3 \mathrm{~d}, 1 \mathrm{w}, 2 \mathrm{w}, 3 \mathrm{w}, 4 \mathrm{w}, 5 \mathrm{w}, 6 \mathrm{w}, 7 \mathrm{w}, 8 \mathrm{w}, 9 \mathrm{w}, 10 \mathrm{w}, 11 \mathrm{w}$, and $12 \mathrm{w}$. The light gray boxes indicate literature reference values, and the gray boxes indicate the control group in our test. $\mathbf{c}$ Blood circulation time of PEG-GNRs analyzed by measuring the remaining Au content in blood. $\mathbf{d}$ The biodistribution of PEG-GNRs in different organs at 12 weeks after treatment. e Histological images of the major organs of the control and experimental animal \#1 (number 1) 12 weeks post-injection with PEG-GNRs. Images were acquired at $200 \times$ magnification 


\section{The preparation and characterization of CTAB-GNRs} and PEG-GNRs

We prepared CTAB-GNRs using a modified version of our previously published seed-mediated approach [54]. The concentration of $\mathrm{Au}$ in $\mathrm{CTAB}-\mathrm{GNRs}$ dispersion was determined via lysis $\left(\mathrm{HNO}_{3}: \mathrm{HCl}: \mathrm{HClO}_{4}=3: 1: 2\right)$ and ICP-AES analysis (SPECTRO ARCOS, Spectro, Germany).

The surface molecule $C T A B$ was then replaced with $\mathrm{mPEG}_{5000}-\mathrm{SH}$. The CTAB-GNRs were purified via centrifugation twice and redispersed in deionized water to a final concentration of $[\mathrm{Au}]$ of $1 \mathrm{mg} / \mathrm{mL}$. Then, $200 \mathrm{mg}$ thiolated-PEG in $32 \mathrm{~mL}$ water was added to the CTABGNRs and the mixture was gently stirred overnight. The molar ratio of PEG-SH and Au was 0.20. Finally, the obtained PEG-GNRs were purified by centrifugation twice.

The CTAB-GNRs and PEG-GNRs were characterized by TEM, AFM, UV-Vis, and zeta potentials as described elsewhere $[4,54]$.

\section{Small animal study}

The study was approved by the ethics committee of the State Key Laboratory of Oral Diseases and conducted in compliance with the animal care and use guidelines of Sichuan University. Male SD rats (6-8 weeks old) were purchased from the Experimental Animals Center of Sichuan Province, China. The rats were categorized into four groups. The control group was injected with normal saline, and the other groups were administered 5 ,

Table 1 Urine test of the control Macaca fascicularis in 12 weeks

\begin{tabular}{|c|c|c|c|c|c|c|c|c|c|c|c|c|}
\hline Date week & GLU mmol/L & $\mathrm{BIL} \mu \mathrm{mol} / \mathrm{L}$ & KET mol/L & SG & BLO Ery/ $\mu \mathrm{L}$ & $\mathrm{pH}$ & PRO g/L & URO $\mu \mathrm{mol} / \mathrm{L}$ & NIT & LEU leu/ $\mu \mathrm{L}$ & Color & Clarity \\
\hline 0 & - & - & 3.9 & 1.01 & - & 7.5 & - & 3.2 & + & - & Yellow & Clear \\
\hline 1 & - & - & - & 1.02 & - & 8.5 & - & 3.2 & - & - & Yellow & Clear \\
\hline 2 & - & - & - & 1.02 & Trace & $\geq 9$ & - & 3.2 & - & - & Yellow & Clear \\
\hline 3 & - & - & - & 1.02 & Trace & $\geq 9$ & - & 3.2 & - & - & Yellow & Clear \\
\hline 4 & - & - & - & 1.02 & - & 8.5 & 0.3 & 3.2 & - & - & Yellow & Clear \\
\hline 5 & - & - & - & 1.02 & - & $\geq 9$ & - & 3.2 & - & - & Yellow & Clear \\
\hline 6 & - & - & - & 1.02 & - & 8.5 & - & 3.2 & - & - & Yellow & Clear \\
\hline 7 & - & - & - & 1.02 & - & $\geq 9$ & - & 3.2 & - & - & Yellow & Clear \\
\hline 8 & - & - & - & 1.02 & - & $\geq 9$ & - & 3.2 & - & - & Yellow & Clear \\
\hline 9 & - & - & - & 1.02 & - & $\geq 9$ & 0.3 & 3.2 & + & - & Yellow & Clear \\
\hline 10 & - & - & - & 1.01 & - & 8.5 & 0.3 & 3.2 & + & - & Yellow & Clear \\
\hline 11 & - & - & - & 1.01 & - & $\geq 9$ & 1 & 3.2 & - & - & Yellow & Clear \\
\hline 12 & - & - & - & 1.02 & - & 8.0 & 1 & 3.2 & - & - & Yellow & Clear \\
\hline
\end{tabular}

GLU Glucose, BIL Bilirubin, KET Ketone, SG Specific Gravity, BLO Occult Blood, PRO Protein, URO Urobilinogen, NIT Nitrite, LEO Leukocyte

Table 2 Urine test of the PEG-GNRs treated Macaca fascicularis (number 1) in 12 weeks

\begin{tabular}{|c|c|c|c|c|c|c|c|c|c|c|c|c|}
\hline Date Week & $\mathrm{GLU} \mathrm{mmol} / \mathrm{L}$ & $\mathrm{BIL} \mu \mathrm{mol} / \mathrm{L}$ & KET $\mathrm{mol} / \mathrm{L}$ & SG & BLO Ery/ $\mu \mathrm{L}$ & $\mathrm{pH}$ & PRO g/L & URO $\mu \mathrm{mol} / \mathrm{L}$ & NIT & LEU leu/ $\mu \mathrm{L}$ & Color & Clarity \\
\hline 0 & - & - & 3.9 & 1.01 & 5 & $\geq 9$ & - & 3.2 & - & - & Yellow & Clear \\
\hline 1 & - & - & - & 1.02 & - & 8.5 & - & 3.2 & - & - & Yellow & Clear \\
\hline 2 & - & - & - & 1.02 & Trace & $\geq 9$ & - & 3.2 & - & - & Yellow & Clear \\
\hline 3 & - & - & - & 1.02 & Trace & 8.5 & - & 3.2 & + & - & Yellow & Clear \\
\hline 4 & - & - & - & 1.02 & - & $\geq 9$ & - & 3.2 & - & - & Yellow & Clear \\
\hline 5 & - & - & - & 1.02 & - & $\geq 9$ & - & 3.2 & - & - & Yellow & Clear \\
\hline 6 & - & - & - & 1.02 & Trace & $\geq 9$ & Trace & 3.2 & Trace & - & Yellow & Clear \\
\hline 7 & - & - & - & 1.02 & - & $\geq 9$ & - & 3.2 & - & - & Yellow & Clear \\
\hline 8 & - & - & - & 1.02 & - & $\geq 9$ & - & 3.2 & - & - & Yellow & Clear \\
\hline 9 & - & - & Trace & 1.02 & - & 8.5 & - & 3.2 & - & - & Yellow & Clear \\
\hline 10 & - & - & - & 1.02 & - & 8.5 & - & 3.2 & - & - & Yellow & Clear \\
\hline 11 & - & - & - & 1.01 & - & 8.5 & - & 3.2 & - & - & Yellow & Clear \\
\hline 12 & - & - & - & 1.02 & - & 8.5 & - & 3.2 & - & - & Yellow & Clear \\
\hline
\end{tabular}


Table 3 Urine test of the PEG-GNRs treated Macaca fascicularis (number 2) in 12 weeks

\begin{tabular}{|c|c|c|c|c|c|c|c|c|c|c|c|c|}
\hline Date Week & $\mathrm{GLU} \mathrm{mmol} / \mathrm{L}$ & $\mathrm{BIL} \mu \mathrm{mol} / \mathrm{L}$ & KET mol/L & SG & 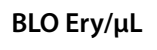 & $\mathrm{pH}$ & PRO g/L & URO $\mu \mathrm{mol} / \mathrm{L}$ & NIT & LEU leu/ $\mu \mathrm{L}$ & Color & Clarity \\
\hline 0 & - & - & - & 1.01 & - & 8.5 & - & 3.2 & - & - & Yellow & Clear \\
\hline 1 & - & - & - & 1.02 & Trace & 7.0 & - & 3.2 & - & - & Yellow & Clear \\
\hline 2 & - & - & - & 1.02 & Trace & 8.5 & - & 3.2 & - & - & Yellow & Clear \\
\hline 3 & - & - & - & 1.02 & Trace & 8.5 & - & 3.2 & - & - & Yellow & Clear \\
\hline 4 & - & - & - & 1.01 & - & $\geq 9$ & Trace & 3.2 & - & - & Yellow & Clear \\
\hline 5 & - & - & - & 1.01 & - & $\geq 9$ & - & 3.2 & - & - & Yellow & Clear \\
\hline 6 & - & - & - & 1.01 & Trace & $\geq 9$ & 1 & 3.2 & - & - & Yellow & Clear \\
\hline 7 & - & - & - & 1.02 & Trace & $\geq 9$ & - & 3.2 & - & - & Yellow & Clear \\
\hline 8 & - & - & - & 1.01 & - & $\geq 9$ & - & 3.2 & - & - & Yellow & Clear \\
\hline 9 & - & - & - & 1.02 & - & 8.5 & - & 3.2 & - & - & Yellow & Clear \\
\hline 10 & - & - & - & 1.01 & - & $\geq 9$ & 0.3 & 3.2 & - & - & Yellow & Clear \\
\hline 11 & - & - & - & 1.01 & Trace & 7.5 & - & 3.2 & - & - & Yellow & Clear \\
\hline 12 & - & - & - & 1.02 & - & 8.5 & 1 & 3.2 & - & - & Yellow & Clear \\
\hline
\end{tabular}

GLU Glucose, BIL Bilirubin, KET Ketone, SG Specific Gravity, BLO Occult Blood, PRO Protein, URO Urobilinogen, NIT Nitrite, LEO Leukocyte

Table 4 Urine test of the PEG-GNRs treated Macaca fascicularis (number 4) in 12 weeks

\begin{tabular}{|c|c|c|c|c|c|c|c|c|c|c|c|c|}
\hline Date Week & GLU mmol/L & $\mathrm{BIL} \mu \mathrm{mol} / \mathrm{L}$ & KET $\mathrm{mol} / \mathrm{L}$ & SG & BLO Ery/ $\mu \mathrm{L}$ & $\mathrm{pH}$ & PRO g/L & URO $\mu \mathrm{mol} / \mathrm{L}$ & NIT & LEU leu/ $\mu \mathrm{L}$ & Color & Clarity \\
\hline 0 & - & - & Trace & 1.01 & - & $\geq 9$ & - & 3.2 & + & - & Yellow & Clear \\
\hline 1 & - & - & - & 1.02 & - & 8.5 & - & 3.2 & - & - & Yellow & Clear \\
\hline 2 & - & - & - & 1.01 & Trace & $\geq 9$ & 1 & 3.2 & - & - & Yellow & Clear \\
\hline 3 & - & - & Trace & 1.01 & - & $\geq 9$ & - & 3.2 & + & - & Yellow & Clear \\
\hline 4 & - & - & - & 1.01 & Trace & $\geq 9$ & 1 & 3.2 & - & - & Yellow & Clear \\
\hline 5 & - & - & - & 1.02 & - & $\geq 9$ & - & 3.2 & - & - & Yellow & Clear \\
\hline 6 & - & - & - & 1.02 & Trace & $\geq 9$ & Trace & 3.2 & Trace & - & Yellow & Clear \\
\hline 7 & - & - & Trace & 1.01 & Trace & $\geq 9$ & 0.3 & 3.2 & - & - & Yellow & Clear \\
\hline 8 & - & - & - & 1.01 & - & 7.5 & 0.3 & 3.2 & - & - & Yellow & Clear \\
\hline 9 & - & - & - & 1.01 & - & $\geq 9$ & - & 3.2 & - & - & Yellow & Clear \\
\hline 10 & - & - & - & 1.02 & 5 & 8.5 & Trace & 3.2 & - & - & Yellow & Clear \\
\hline 11 & - & - & - & 1.01 & Trace & 8.5 & - & 3.2 & - & - & Yellow & Clear \\
\hline 12 & - & - & - & 1.01 & - & 8.5 & - & 3.2 & - & - & Yellow & Clear \\
\hline
\end{tabular}

GLU Glucose, BIL Bilirubin, KET Ketone, SG Specific Gravity, BLO Occult Blood, PRO Protein, URO Urobilinogen, NIT Nitrite, LEO Leukocyte

10 , and $20 \mathrm{mg} / \mathrm{kg}$ of PEG-GNRs by tail vein injection. At $0.25,0.75,1,2,6,12,24$, and $48 \mathrm{~h}$, tail vein blood samples were obtained to determine the concentrations of $\mathrm{Au}$ in the blood. Blood markers were assessed at 3 days and at 1 week and 2 weeks post-injection. The major organs were lysed, and PEG-GNRs was detected by ICPAES. After 2 weeks, all rats were sacrificed and the major organs were collected for $\mathrm{H} \& \mathrm{E}$ staining.

\section{Macaca fascicularis study}

The M. fascicularis study was supported by West ChinaFrontier Pharma Tech Co. (WCFP), National Chengdu Center for Safety Evaluation of Drugs (NCCSED), China. The protocol was reviewed and approved by the ethics committee of NCCSED. Four adult male $M$. fascicularis (age 3-4 years, body mass was $\sim 4.0 \mathrm{~kg}$ ) were used in the toxicity study ( 3 were treated with PEG-GNRs and 1 was considered the control). Animals were individually housed in stainless steel cages and fed a commercial monkey diet from local supplies. Water was available ad libitum. The professional research staff regularly inspected the animals twice a day.

The animals received $1.0 \mathrm{mg} / \mathrm{kg}$ of PEG-GNRs by intravenous drip over $30 \mathrm{~min}$. The control animal was administered normal saline. Peripheral blood samples were collected at designated times to determine the blood clearance of PEG-GNRs and other parameters. The blood markers analysis was conducted on a Siemens Advia 2120 and a Roche Cobas 6000-C501. Every week, 3-5 mL of urine was collected for urinalysis on a Siemens Clinitek 


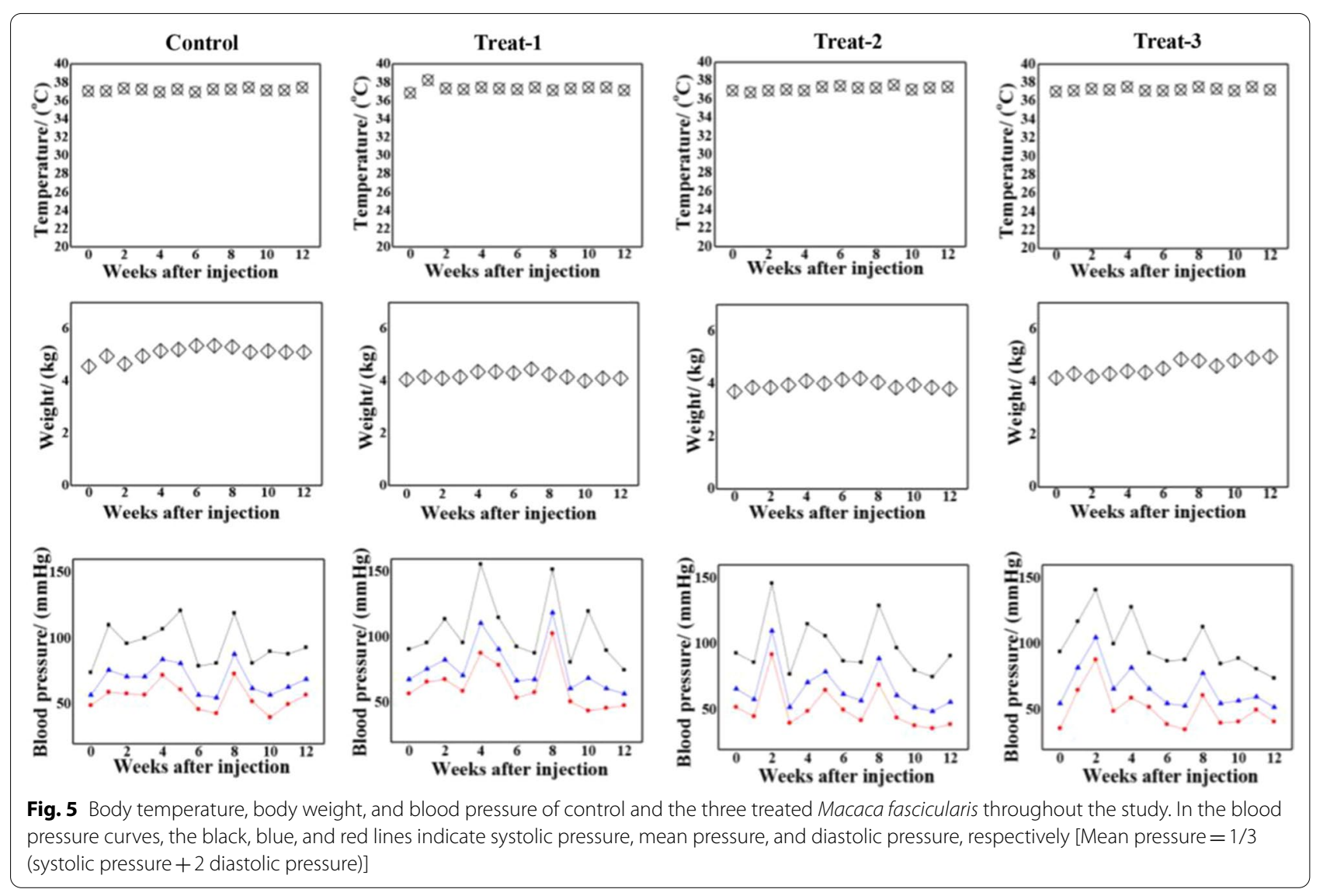

Status machine. Body mass, body temperature, blood pressure, appearance, and exploratory behavior of the Macaca fascicularis were simultaneously recorded. After 12 weeks, the animals were sacrificed for tissue analysis. The heart, liver, spleen, lungs, kidneys, brain, lymph, intestine, skeleton muscle, and skin were collected. The organs were lysed to measure the amount of Au by ICPAES. Meanwhile, the remaining tissues were fixed for obtaining H\&E stained histological sections and examined with an Olympus BX60 microscope.

\section{Conclusions}

This study broadened the study of systemic PEG-GNRs toxicity research from rodents to non-human primates in a preclinical test. We detected a negligible immune response in the blood marker analysis of $M$. fascicularis. This immune response quickly disappeared suggesting a normal immune reaction to any foreign body. All the animals appeared healthy during the 12-week study period, supported by normal blood, urine, body temperature, body masses, and blood pressure levels. Biodistribution analysis showed that PEG-GNRs primarily accumulated in the RES system, but histological analysis revealed no apparent damage to these organs. Overall, the PEGGNRs showed excellent biocompatibility in both rats and $M$. fascicularis at clinically relevant doses. The nonhuman primate safety evaluation of PEG-GNRs showed great potential for human clinical application. More detailed, long-term investigations of GNRs in higher mammals are required.

\section{Supplementary Information}

The online version contains supplementary material available at https://doi. org/10.1186/s12951-021-00941-1.

Additional file 1: Figure S1. The bio-distribution at 3 days, 1 week, and 2 weeks after systemic administration of (a) $5 \mathrm{mg} / \mathrm{kg}$ and (b) $20 \mathrm{mg} / \mathrm{kg}$ PEG-GNRs in rats. Figure S2 H\&E staining photographs of major organs in rats at 2 weeks after treatment. Images were acquired at $200 \times$ magnification. Figure S3. Blood markers test for Macaca fascicularis treated with PEG-GNRs. Figure S4. Histological images of major organs of the control and Macaca fascicularis (number 1) post-injection at 12 weeks. Images were acquired at $200 \times$ magnification. Figure S5. Histological images of the major organs of Macaca fascicularis number 2 and number 3 postinjection at 12 weeks.

\section{Acknowledgements}

Not applicable. 


\section{Authors' contributions}

$J \mathrm{~L}, T T$, and $Y L$ conceived the study and participated in preparing the experiment design. JL, TT, SS, and XX performed the experiments. JL wrote the manuscript. $Y Z, S P, J X$ and $Y L$ revised the manuscript and provided the guidance on the paper submission. All the authors read and approved the final manuscript.

\section{Funding}

This study was supported by National Key R\&D Program of China (2019YFA01 10600), the National Natural Science Foundation of China (81970916, 81671031, 31972925, 81870746), and Sichuan Science and Technology Program (2020YJ0065).

\section{Availability of data and materials}

All data are available in the main manuscript and supplementary information.

\section{Declarations}

\section{Ethics approval and consent to participate}

The rat experimental procedure was approved by the ethics committee of the State Key Laboratory of Oral Diseases and conducted in compliance with the animal care and use guidelines of Sichuan University. The Macaca fascicularis study was supported by West China-Frontier Pharma Tech (WCFP), National Chengdu Center for Safety Evaluation of Drugs (NCCSED), China. The protocol was reviewed and approved by the ethics committee of NCCSED.

\section{Consent for publication}

Not applicable.

\section{Competing interests}

The authors declare no competing interests.

\section{Author details}

${ }^{1}$ State Key Laboratory of Oral Diseases, National Clinical Research Centre for Oral Diseases, West China Hospital of Stomatology, Sichuan University, Chengdu 610041, China. ${ }^{2}$ Department of Oral and Maxillofacial Surgery, The Affiliated Stomatology Hospital of Southwest Medical University, Luzhou 646000, China. ${ }^{3}$ Division of Physical Biology, CAS Key Laboratory of Interfacial Physics and Technology, Shanghai Synchrotron Radiation Facility, Shanghai Institute of Applied Physics, Chinese Academy of Sciences, Shanghai 201800, China. ${ }^{4}$ Zhangjiang Laboratory, Shanghai Advanced Research Institute, Chinese Academy of Sciences, Shanghai 201210, China. ${ }^{5}$ College of Biomedical Engineering, Sichuan University, Chengdu 610041, China.

Received: 25 April 2021 Accepted: 21 June 2021

Published online: 30 June 2021

\section{References}

1. Rastinehad AR, Anastos H, Wajswol E, Winoker JS, Sfakianos JP, Doppalapudi SK, Carrick MR, Knauer CJ, Taouli B, Lewis SC, Tewari AK, Schwartz JA, Canfield SE, George AK, West JL, Halas NJ. Gold nanoshell-localized photothermal ablation of prostate tumors in a clinical pilot device study. Proc Natl Acad Sci USA. 2019;116:18590-6.

2. Jana NR, Gearheart L, Murphy CJ. Wet chemical synthesis of high aspect ratio cylindrical gold nanorods. J Phys Chem B. 2001;105:4065-7.

3. Lohse SE, Murphy CJ. The quest for shape control: a history of gold nanorod synthesis. Chem Mater. 2013;25(8):1250-61.

4. Jia YP, Shi K, Liao JF, Peng JR, Hao Y, Qu Y, Chen LJ, Liu L, Yuan X, Qian ZY, Wei XW. Effects of cetyltrimethylammonium bromide on the toxicity of gold nanorods both in vitro and in vivo: molecular origin of cytotoxicity and inflammation. Small Methods. 2020;4:1900799.

5. Guo H, Yi S, Feng K, Xia Y, Qu X, Wan F, Chen L, Zhang C. In situ formation of metal organic framework onto gold nanorods, mesoposous silica with functional integration for targted theranostics. Chem Eng J. 2021;403:126432.

6. Médard G, Papageorgiou AC. Surface anchors target golden bullet. Nat Chem. 2019;11:20-2.
7. Zhao Y-Q, Sun Y, Zhang Y, Ding X, Zhao N, Yu B, Zhao H, Duan S, Xu F-J. Well-defined gold nanorod/polymer hybrid coating with inherent antifouling and photothermal bactericidal properties for treating an infected hernia. ACS Nano. 2020;14:2265-75.

8. Uson L, Sebastian V, Arruebo M, Santamaria J. Continuous microfluidic synthesis and functionalization of gold nanorods. Chem Eng J. 2016:285:286-92.

9. Chen G, Wang Y, Wu P, Zhou Y, Yu F, Zhu C, Li Z, Huang Y, Wang K, Li J, Sun M, Oupicky D. Reversibly stabilized polycation nanoparticles for combination treatment of early- and late-stage metastatic breast cancer. ACS Nano. 2018;12:6620-36.

10. Li Z, Zhu L, Sun H, Shen Y, Hu D, Wu W, Wang Y, Qian C, Sun M. Fluorine assembly nanocluster breaks the shackles of immunosuppression to turn the cold tumor hot. Proc Natl Acad Sci USA. 2020;117:32962-9.

11. González-Rubio G, Díaz-Núñez P, Rivera A, Prada A, Tardajos G, GonzálezIzquierdo J, Bañares L, Llombart P, Macdowell LG, Palafox MAL, Liz-Marzán M, Peña-Rodríguez O, Guerrero-Martínez A. Femtosecond laser reshaping yields gold nanorods with ultranarrow surface plasmon resonances. Science. 2017;358:640-4.

12. Li N, Zhao P, Astruc D. Anisotropic gold nanoparticles: synthesis, properties, applications, and toxicity. Angew Chem Int Ed. 2014;53:1756-89.

13. Wawra SE, Pflug L, Thajudeen T, Kryschi C, Stingl M, Peukert W. Determination of two-dimensional distributions of gold nanorods by multiwavelength analytical ultracentrifugation. Nat Commun. 2018;9:4898.

14. Liu T, Tong L, Lv N, Ge X, Fu Q, Gao S, Ma Q, Song J. Two-stage size decrease and enhanced photoacoustic performance of stimuli-responsive polymer-gold nanorod assembly for increased tumor penetration. Adv Funct Mater. 2019;16:1806429.

15. Thakor AS, Jokerst J, Zavaleta C, Massoud TF, Gambhir SS. Gold nanoparticles: a revival in precious metal administration to patients. Nano Lett. 2011;11:4029-36.

16. Li B, Wang Y, He J. Gold nanorods-based smart nanoplatforms for synergic thermotherapy and chemotherapy of tumor metastasis. ACS Appl Mater Interfaces. 2019;11:7800-11.

17. Lippok N, Villiger M, Albanese A, Meijer EFJ, Chuang K, Padera TP, Bhatia SN, Bouma BE. Depolarization signatures map gold nanorods within biological tissue. Nat Photonics. 2017;11:583-8.

18. Wang WN, Pei P, Chu ZY, Chen BJ, Qian HS, Zha ZB, Zhou W, Liu T, Shao $\mathrm{M}$, Wang $\mathrm{H}_{\text {. }} \mathrm{Bi}_{2} \mathrm{~S}_{3}$ coated Au nanorods for enhanced photodynamic and photothermal antibacterial activities under NIR light. Chem Eng J. 2020;397:125488.

19. Lee C, Lim K, Kim SS, Thien LX, Lee ES, Oh KT, Choi H-G, Youn YS. Chlorellagold nanorods hydrogels generating photosynthesis-drived oxygen and mild heat for the treatment of hypoxic breast cancer. J Control Rel. 2019;294:77-90.

20. Yang Z, Du Y, Sun Q, Peng Y, Wang R, Zhou Y, Wang Y, Zhang C, Qi X. Alleviating potentiates synchronous multimodal imaging and phototherapy for glioma. ACS Nano. 2020;14:6191-212.

21. Zijlstra P, Paulo PMR, Orrit M. Optical detection of single non-absorbing molecules using the surface plasmon resonance of a gold nanorod. Nat Nanotech. 2012;7:379-82.

22. Chen Y-S, Zhao Y, Yoon SJ, Gambhir SS, Emelianov S. Gold nanorods for photoacoustic molecular imaging in the second near-infrared optical window. Nat Nanotech. 2019;14:465-72.

23. Zijlstra P, Chon JWM, Gu M. Five-dimensional optical recording mediated by surface plasmons in gold nanorods. Nature. 2009;459:410-3.

24. Huang QQ, Zou Y, Zhong SC, Yang X, Li J, Huang WX, Zhu HF, Cheng C, Ding MM, Zhu LG, Shi QW. Silica-coated gold nanorods with high photothermal efficiency and biocompatibility as a contrast agent for in vitro terahertz imaging. J Biomed Nanotechnol. 2019;15:910-20.

25. Xu W, Qian J, Hou G, Suo A, Wang Y, Wang J, Sun T, Yang M, Wan X, Yao Y. Hyaluronic acid-functionalized gold nanorods with $\mathrm{pH} / \mathrm{NIR}$ dual-responsive drug release for synergetic targeted photothermal chemotherapy of breast cancer. ACS Appl Mater Interfaces. 2017;9:36533-47.

26. Zhang L, Su H, Cai J, Cheng D, Ma Y, Zhang J, Zhou C, Liu S, Shi H, Zhang $Y$, Zhang C. Multifunctional platform for tumor angiogenesis-targeted chemo-thermal therapy using polydopamine-coated gold nanorods. ACS Nano. 2016;10:10404-17.

27. Volsi AL, Scialabba C, Vetri V, Cavallaro G, Licciardi M, Giammona G. Nearinfrared light responsive folate targeted gold nanorods for combined 
photothermal-chemotherapy of osteosarcoma. ACS Appl Mater Interfaces. 2017;9:14453-69.

28. Salem AK, Searson PC, Leong KW. Multifunctioal nanorods for gene delivery. Nat Mater. 2003;2:668-71.

29. Min KH, Kim Y-H, Wang Z, Kim J, Kim JS, Kim SH, Kim K, Kwon IC, Kiesewetter DO, Chen X. Engineered Zn(II)-dipicolylamine-gold nanorod provides effective prostate cancer treatment by combining siRNA delivery and photothermal therapy. Theranostics. 2017;7:4240-54.

30. Baaske MD, Foreman MR, Vollmer F. Single-molecule nucleic acid interactions monitored on a label-free microcavity biosensor platform. Nat Nanotech. 2014;9:933-9.

31. Qin $L, H e X$, Chen $L$, Zhang Y. Turn-on fluorescent sensing of glutathione $S$-transferase at near-infrared region based on FRET between gold nanoclusters and gold nanorods. ACS Appl Mater Interfaces. 2015;7:5965-71.

32. Luan J, Seth A, Gupta R, Wang Z, Rathi P, Cao S, Derami HG, Tang R, Xu B, Achilefu S, Morrissey JJ, Sigamaneni S. Ultrabright fluorescent nanoscale labels for the gemtomolar detection of analyates with standard bioassays. Nat Biomed Eng. 2020;4:518-30.

33. Khlebtsov N, Dykman L. Biodistribution and toxicity of engineered gold nanoparticles: a review of in vitro and in vivo studies. Chem Soc Rev. 2011;40:1647-71.

34. Ye L, Yong K-T, Liu L, Roy I, Hu R, Zhu J, Cai H, Law W-C, Liu J, Wang K, Liu J, Liu Y, Hu Y, Zhang X, Swihart MT, Prasad PN. A pilot study in non-human primates shows no adverse response to intravenous injection of quantum dots. Nat Nanotech. 2012;7:453-8.

35. Yong K-T, Law W-C, Hu R, Ye L, Liu L, Swihart MT, Prasad PN. Nanotoxicity assessment of quantum dots: from cellular to primate studies. Chem Soc Rev. 2013:42:1236-50.

36. Liu J, Erogbogbo F, Yong K-T, Ye L, Liu J, Hu R, Chen H, Hu Y, Yang Y, Yang J, Roy I, Karker NA, Swihart MT, Prasad PN. Assessing clinical prospects of silicon quantum dots: studies in mice and monkeys. ACS Nano. 2013;7:7303-10

37. Moore L, Yang J, Lan TTH, Osawa E, Lee D-K, Johnson WD, Xi J, Chow EK-H, Ho D. Biocompatibility assessment of detonation nanodiamond in non-huamn primates and rats using histological, hematologic, and urine analysis. ACS Nano. 2016;10:7385-400.

38. Lin Y, Zhang Y, Li J, Kong H, Yan Q, Zhang J, Li W, Ren N, Cui Y, Zhang T, Cai X, Li Q, Li A, Shi J, Wang L, Zhu Y, Fan C. Blood exposure to graphene oxide may cause anaphylatic death in non-human primates. Nano Today. 2020;35:100922.

39. Lu Y, Xu Y-J, Zhang G, Ling D, Wang M, Zhou Y, Wu Y-D, Wu T, Hackett MJ, Kim BH, Chang H, Kim J, Hu X-T, Dong L, Lee N, Li F, He J-C, Zhang L, Wen $H-\mathrm{Q}$, Yang B, Choi SH, Hyeon T, Zou D-H. Iron oxide nanoclusters for $\mathrm{T}_{1}$ magnetic resonance imaging of non-human primates. Nat Biomed Eng. 2017;1:637-43.

40. Lankveld D, Rayavarapu R, Krystec P, Oomen AG, Verharen HW, Leeuwen TG, Jong W, Manohar S. Blood clearance and tissue distribution of pegylated and non-pegylated gold nanorods after intravenous administration in rats. Nanomed. 2011;6:339-49.
41. Dam DHM, Culver KSB, Kandela I, Lee RC, Chandra K, Lee H, Mantis C, Ugolkov A, Mazar AP, Odom TW. Biodistribution and in vivo toxicity of aptamer-loaded gold nanostars. Nanomed Nanotechnol. 2015;11:671-9.

42. Akiyama Y, Mori T, Katayama Y, Niidome T. The effects of PEG grafting level and injection dose on gold nanorod biodistribution in the tumor-bearing mice. J Control Rel. 2009;139:81-4.

43. Zhang Z, Wang J, Nie X, Wen T, Ji Y, Wu X, Zhao Y, Chen C. Near infrared laser induced targeted cancer therapy using thermo-responsive polymer encapsulated gold nanorods. J Am Chem Soc. 2014;136:7317-26.

44. Niidome T, Yamagata M, Okamoto Y, Akiyama Y, Takahashi H, Kawano T, Katayama Y, Niidome Y. PEG-modified gold nanorods with a stealth character for in vivo applications. J Control Rel. 2006;114:343-7.

45. Manohar S, Ungureanu C, Leeuwen TGV. Gold nanorods as molecular contrast agents in photoacoustic imaging: the promises and the caveats. Contrast Media Mol Imaging. 2011;6:389-400.

46. Allen SD, Liu Y-G, Bobbala S, Cai L, Hecker PI, Temel R, Scott EA. Nano Res. 2018;11:5689-703.

47. Zhao L, Yuan W, Ang CY, Qu Q, Dai Y, Gao Y, Luo Z, Wang J, Chen H, Li M, Li F, Zhao Y. Silica-polymer hybrid with self-assembled PEG corona excreted rapidly via a hepatobiliary route. Adv Funct Mater. 2016;26:3036-47.

48. Xu J, Yu M, Peng C, Carter P, Tian J, Ning X, Zhou Q, Tu Q, Zhang G, Dao A, Jiang X, Kapur P, Hsieh J-T, Zhou X, Liu P, Zheng J. Dose dependencies and biocompatibility of renal clearable gold nanoparticles: from mice to non-huamn primates. Angew Chem Int Ed. 2018;57:226-71.

49. Zhang Q, Lin S, Wang L, Peng S, Tian T, Li S, Xiao J, Lin Y. Tetrahedral framework nucleic acids act as antioxidants in acute kidney injury treatment. Chem Eng J. 2021;127426.

50. Zhou H, Oh S, Kim JE, Zou F, Hwang DY, Lee J. In vivo study of spiky $\mathrm{Fe}_{3} \mathrm{O}_{4} @$ Au nanoparticles with different branch lengths: biodistribution, clearance, and biocompatibility in mice. ACS Appl Bio Mater. 2019;2:163-70.

51. Ali MRK, Wu Y, El-Sayed MA. Gold-nanoparticle-assisted plasmonic photothermal therapy advances toward clinical application. J Phys Chem C. 2019;123:15375-93

52. Ali MR, Rahman MA, Wu Y, Han T, Peng $X$, Mackey MA, Wang DHJ, Shin ZG, Chen H, Xiao R, Tang WY, Shin DM, El-Sayed MA. Efficacy, long-term toxicity, and mechanistic studies of gold nanorods photothermal therapy of cancer in xenograft mice. Proc Natl Acad Sci USA. 2017;114:E3110-8.

53. Huang H, Feng W, Chen Y, Shi J. Inorganic nanoparticles in clinical and translation. Nano Today. 2020;35:100972.

54. Liao JF, Li WT, Peng JR, Yang Q, Li H, Wei YQ, Zhang XN, Qian ZY. Combined cancer photothermal-chemotherapy based on doxorubincin/gold nanorod-loaded polymersomes. Theranostics. 2015;5:345-56.

\section{Publisher's Note}

Springer Nature remains neutral with regard to jurisdictional claims in published maps and institutional affiliations.

\footnotetext{
Ready to submit your research? Choose BMC and benefit from:

- fast, convenient online submission

- thorough peer review by experienced researchers in your field

- rapid publication on acceptance

- support for research data, including large and complex data types

- gold Open Access which fosters wider collaboration and increased citations

- maximum visibility for your research: over $100 \mathrm{M}$ website views per year
}

At BMC, research is always in progress.

Learn more biomedcentral.com/submissions 